\title{
Potencial económico de cuatro especies aromáticas promisorias para producir aceites esenciales en Colombia
}

\section{The economic potential of four promising aromatic species for the production of essential oils in Colombia}
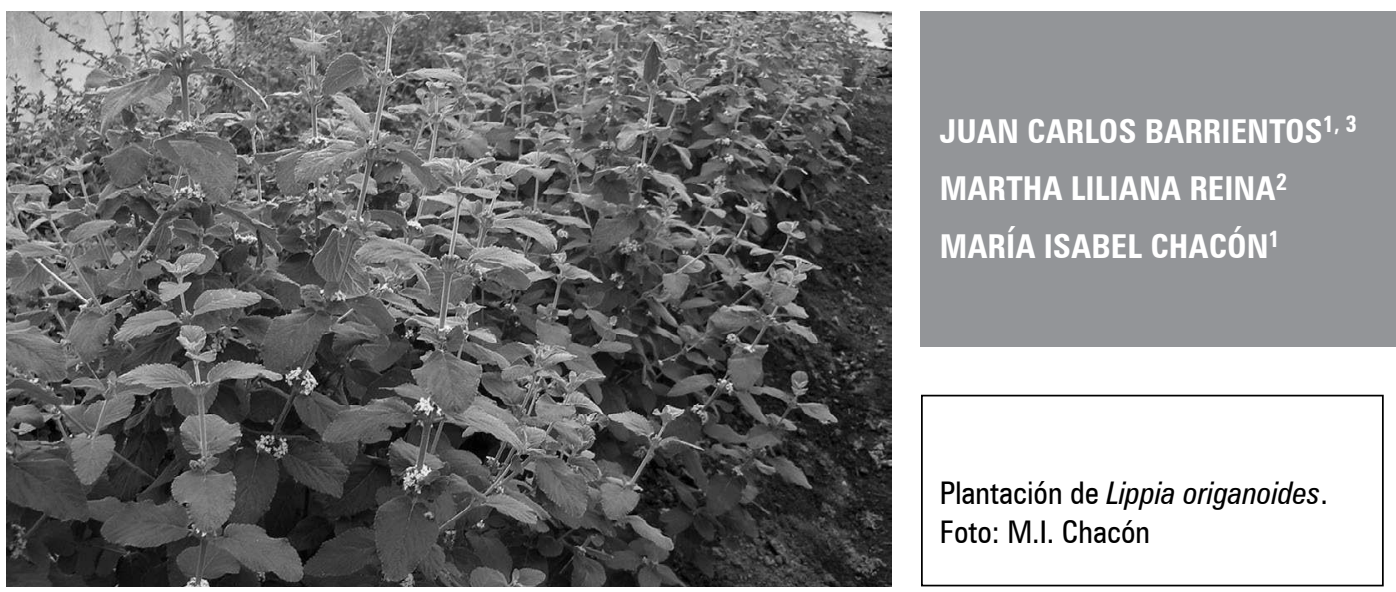

\section{RESUMEN}

En el marco del proyecto de investigación "Evaluación fenotípica, química y agronómica de especies nativas y foráneas promisorias por calidad de aceites esenciales y con uso potencial en el sector agrícola" fueron seleccionadas las especies: Lippia alba, Lippia origanoides, Tagetes caracasana y Tagetes zypaquirensis por ser promisorias en calidad de aceites esenciales con uso potencial en el sector agrícola. Sin embargo, su potencial comercial también depende de su viabilidad económica. En ese sentido la investigación tuvo como objetivo determinar el potencial económico de las mencionadas especies para producir aceites esenciales en Colombia. Para ello se procedió a analizar, a partir de información primaria y secundaria, la estructura de costos para producción y transformación comercial, los precios e ingresos por producto en fresco y aceites esenciales, y las rentabilidades. El análisis económico tomó como referencia dos sistemas de producción y tres escenarios geográficos. La rentabilidad se analizó en términos de valor actual neto (VAN), relación beneficio-costo (R B/C) y tasa interna de retorno (TIR). Los resultados evidencian que todos los cultivos bajo estudio, excepto el de las Lippia bajo invernadero, tienen rentabilidades altas; aunque sus costos de producción son relativamente elevados. El rendimiento de aceite de Lippia fue de aproximadamente 1\%, especialmente en zonas cálidas; y el de Tagetes fue menor a $0,5 \%$. La materia prima es el rubro más costoso en industria ( $>90 \%)$. Para una producción constante de aceite esencial en una unidad industrial de $600 \mathrm{~kg}$ de capacidad por ciclo se requiere entre 7 y 16 ha de cultivo. Las Lippia, por su razonable rendimiento de aceite y precio, son potencialmente rentables; los Tagetes, por el contrario, aún no.

Facultad de Agronomía, Departamento de Agronomía, Universidad Nacional de Colombia, Bogotá (Colombia).

2 Facultad de Agronomía, Programa de Maestría en Ciencias Agrarias, Línea Desarrollo Empresarial Agropecuario, Universidad Nacional de Colombia, Bogotá (Colombia).

3 Autor para correspondencia. jcbarrientosf@unal.edu.co 


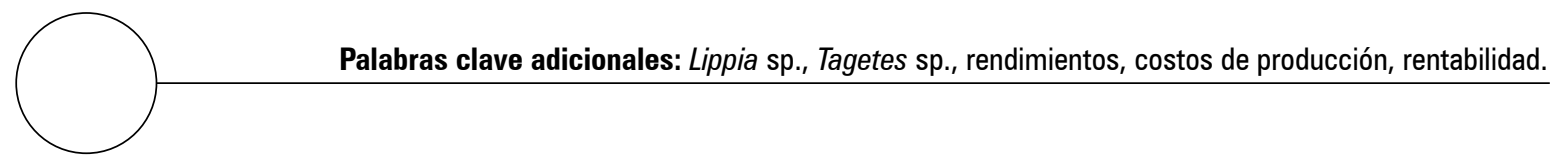

\section{ABSTRACT}

As part of the research project "Phenotypic, chemical, and agronomic assessment of promising native and foreign species based on the quality of their essential oils and potential use in agriculture", the following species were selected: Lippia alba, Lippia origanoides, Tagetes caracasana, and Tagetes zypaquirensis, as promising essential oil species with potential uses in agriculture. However, their commercial potential also depends on their economic viability. In that sense, this research aimed to determine the economic potential of the above mentioned species to produce essential oils in Colombia. Based on primary and secondary information, the cost structure for commercial production and processing, prices and revenues of fresh products and essential oils, and yields were analyzed. The economic analysis took two production systems and three geographic scenarios as the reference. The profitability was analyzed in terms of net present value (NPV), benefit/cost ratio $(B / C R)$ and internal rate of return (IRR). The results show that all the crops under the study, except the greenhouse Lippia, had high yields, although their production costs are relatively high. The oil yield of Lippia is approximately $1 \%$, especially in warm areas, and for Tagetes, it is less than $0.5 \%$. The raw material is the most costly item in the oil industry $(>90 \%)$. For a constant production of essential oils in an industrial unit of a $600 \mathrm{~kg}$ capacity per cycle, between 7 and 16 ha of cultivation are needed. Lippia plants are potentially profitable because of their reasonable oil yield and price, Tagetes plants, however, are not.

Additional key words: Lippia sp., Tagetes sp., yields, production costs, profitability.

Fecha de recepción: 16-08-2012

Aprobado para publicación: 28-11-2012

INTRODUCCIÓN

Las plantas aromáticas contienen cantidades apreciables de fitoquímicos (fenoles, sus derivados y otros), que se encuentran en toda la planta o partes de ella (Bareño y Clavijo, 2006; Pacheco y Pohlan, 2006). En Colombia, según Díaz (2003), hay más de 200 especies bajo la denominación de plantas aromáticas. De acuerdo con el mismo autor, entre $50 \%$ y $60 \%$ de estas plantas corresponden a especies nativas y $20 \%$ a $40 \%$ a especies naturalizadas. La superficie de cultivo de aromáticas en Colombia fue para el año 2008 poco más de 1.200 ha con cerca de 33.000 t de producción (Ministerio de Agricultura y Desarrollo Rural et al., 2008).
La mayor parte de la producción de aromáticas proviene de especies comerciales destinadas a la exportación principalmente; entre ellas se mencionan: albahaca, tomillo, romero, cebollín, orégano, menta, estragón, mejorana, salvia, caléndula, manzanilla y hierbabuena (Bareño y Clavijo, 2006; Díaz, 2006; Repetto, 2005). En el mercado nacional la oferta se destina principalmente al consumo en fresco, ya sea como condimento o como medicamento de uso tradicional, pero también como materia prima o básica para laboratorios y tiendas naturistas que le dan al producto un valor agregado (Colombian National Press, 2006), transformándolas en productos 
medicinales o cosméticos (Fundacofan, 2004). En Colombia, la demanda de plantas aromáticas para consumo en fresco es bastante reducida, y para producción de aceites esenciales aún más. Sin embargo, la industria de aceites esenciales tiene un gran potencial, por la gran diversidad de especies y zonas de producción existentes en el territorio.

El 65\% del volumen de aceites esenciales del mercado proviene de especies cultivadas y solo un $1 \%$ de especies silvestres ( $2 \%$ en valores monetarios); y un 30\% proviene de árboles como pinos, cedros, sándalo, canelo, clavo, ylang-ylang, eucaliptos, enebro, etc. (Bandoni, 2002). En este contexto se ha desarrollado, y se está ejecutando, el programa de investigación denominado "Aprovechamiento de aceites esenciales de especies nativas y foráneas promisorias para uso agrícola en Colombia", financiado por el Ministerio de Agricultura y Desarrollo Rural y ejecutado por la Universidad Nacional de Colombia, sede Bogotá y por la Universidad de Cundinamarca, cuyo objetivo es incrementar la oferta colombiana, tanto en cantidad como en calidad, de aceites esenciales en el mercado nacional e internacional. Así, en el marco de uno de sus proyectos denominado "Evaluación fenotípica, química y agronómica de especies nativas y foráneas promisorias por calidad de aceites esenciales y con uso potencial en el sector agrícola" se ha procedido a recolectar en campo 60 especies: 35 de la familia Asteraceae, 18 de la familia Labiatae y 7 de la familia Verbenaceae. De todas ellas, 17 resultaron seleccionadas por su contenido de aceites esenciales, de las que finalmente cuatro especies fueron seleccionadas por su tipo y contenido de aceite. Las especies en mención son: Lippia alba, Lippia origanoides, Tagetes caracasana y Tagetes zypaquirensis. Estas especies se encuentran a la venta en algunas plazas de mercado de ciudades pequeñas aledañas a las zonas de producción, y de grandes como Bogotá, Medellín y Pereira. Dado el poco conocimiento de los consumidores sobre las bondades de estas especies en el campo de la salud y la medicina tradicional, su demanda es baja, alrededor de $5 \mathrm{t}$ año-1. Por otro lado, la demanda de estas plantas para la producción de aceites esenciales es casi nula (Barrientos y Reina, 2012).

Las especies bajo estudio se consideran potenciales para agricultura e industria por las siguientes razones: (1) estas contienen cantidades apreciables de aceites esenciales, (2) Colombia importa aceites esenciales casi ocho veces más de lo que exporta convirtiéndose en un mercado muy interesante (Biocomercio Sostenible, 2003), y (3) el cultivo de estas especies puede constituirse en una alternativa económica para los pequeños agricultores de las zonas productoras y para las pequeñas agroindustrias.

Dado que estas especies son poco cultivadas, no industrializadas y muy poco conocidas por la mayoría de los consumidores de aromáticas, se dispone de muy poca información técnica y económica sobre ellas. En ese sentido, en un esfuerzo por estimar información económica que sirva de base para posteriores trabajos, esta investigación plantea como objetivo general: evaluar el potencial económico de las cuatro especies aromáticas promisorias para producir aceites esenciales en Colombia. Como objetivos específicos tiene: (1) determinar los costos e ingresos de la producción de material fresco de cada especie; (2) determinar la rentabilidad del cultivo de cada especie; (3) determinar los costos e ingresos de la producción de aceite esencial de cada especie; y (4) determinar la rentabilidad de la producción de aceite esencial de cada especie. Esta investigación permitirá luego hacer una proyección de mercado para determinar su viabilidad comercial.

\section{METODOLOGÍA}

Esta investigación es de tipo no experimental, aplicada, principalmente descriptiva, pero también causal, y eminentemente cuantitativa. El trabajo se realizó en el marco geográfico de dos escenarios de estudio principalmente: (1) se establecieron cultivos de ensayo de las cuatro especies en inverna- 
deros de la Facultad de Agronomía de la Universidad Nacional de Colombia (UNAL) en Bogotá, ubicados a $2.630 \mathrm{msnm}$, con una temperatura interna promedio de $16^{\circ} \mathrm{C}$ y (2) la granja "La Esperanza" de propiedad de la Universidad de Cundinamarca localizada en Guavio Bajo (región Sumapaz) del municipio Fusagasugá (Cundinamarca) ubicada a $1.726 \mathrm{msnm}$, con una temperatura promedio de $20^{\circ} \mathrm{C}$ (clima templado-cálido), donde se establecieron cultivos de ensayo de las dos Lippia y Tagetes caracasana a cielo abierto. Un tercer escenario, de donde solo se tienen datos de rendimiento de los aceites, es de la Facultad de Ciencias Agropecuarias (UNAL), sede Palmira, lugar ubicado a $1.001 \mathrm{msnm}$, con una temperatura promedio de $23^{\circ} \mathrm{C}$ (clima cálido).

La investigación inició en agosto de 2011 y se extendió hasta febrero de 2012. Primero se realizó una revisión de literatura concerniente al tema. La recolección de la información primaria (encargados de los ensayos en Bogotá y Fusagasugá, comercializadores de plantas aromáticas de las plazas de mercado Samper Mendoza y Paloquemao en Bogotá, personal técnico de la empresa Fresh Andina y de la empresa Green Andina e investigadores de las dos universidades participantes) se realizó a través de entrevistas personales, en las que se utilizó un cuestionario semiestructurado con preguntas abiertas. También se hicieron observaciones directas en parcelas de ensayo, mercados de aromáticas, empresas productoras e industrias procesadoras de aromáticas, cuya información se recogió en una libreta de campo. Para el análisis, la información de establecimiento, manejo y cosecha del cultivo que se utilizó, por su similitud, fue la misma para las dos Lippia y para los dos Tagetes.

Para el análisis económico se consideró un emprendimiento de producción y transformación de hierbas aromáticas y aceites esenciales con un horizonte de 10 años. Las variables que se tomaron fueron: costos de producción, ingresos y rentabilidad, para la comparación de dos sistemas (escenarios) de producción, uno bajo invernadero y otro a cielo abierto. El costeo de los factores de producción fue directo. Debido a la similitud de manejo agronómico de las especies bajo estudio, se asumieron los mismos costos de cultivo tanto para las Lippia (tablas 2 y 3 ) como para los Tagetes (tablas 4 y 5). Para el cálculo de ingresos y rentabilidad, tanto del cultivo como de la obtención de aceites, se han asumido valores promedio de rendimientos y precios. La información técnica de cultivo provino de los ensayos realizados tanto en Bogotá como en Fusagasugá como se muestran en la tabla 1. Allí se destacan la diferencia de densidades, número de cosechas y producción de Tagetes y Lippia. Para el cálculo de la rentabilidad se han considerado una tasa de descuento del 14\% y un flujo de caja de 10 años basado en el tiempo de vida útil de un invernadero.

\section{Tabla 1. Información técnica sobre el cultivo de Lippia y de Tagetes.}

\begin{tabular}{|l|c|c|c|c|c|}
\hline $\begin{array}{c}\text { Especie, sistema de } \\
\text { cultivo y lugar }\end{array}$ & $\begin{array}{c}\text { Distancia de } \\
\text { siembra (m) }\end{array}$ & $\begin{array}{c}\text { Densidad } \\
\text { (plantas/ha) }\end{array}$ & $\begin{array}{c}\text { Número de } \\
\text { cosechas por año }\end{array}$ & $\begin{array}{c}\text { Producción por } \\
\text { cosecha } \\
\text { (g/planta) }\end{array}$ & $\begin{array}{c}\text { Rendimiento } \\
\text { anual }\left(\mathrm{kg} / \mathrm{ha}^{-1}\right)\end{array}$ \\
\hline $\begin{array}{l}\text { Lippia bajo invernadero } \\
\text { (Bogotá) }\end{array}$ & $1 \times 0,5$ & 20.000 & 4 & 300 & 24.000 \\
\hline $\begin{array}{l}\text { Lippia a cielo abierto } \\
\text { (Fusagasugá) }\end{array}$ & $1 \times 0,5$ & 20.000 & 3 & 250 & 18.000 \\
\hline $\begin{array}{l}\text { Tagetes bajo } \\
\text { invernadero (Bogotá) }\end{array}$ & $0,8 \times 0,3$ & 41.667 & 4 & 250 & 42.000 \\
\hline $\begin{array}{l}\text { Tagetes a cielo abierto } \\
\text { (Fusagasugá) }\end{array}$ & $0,8 \times 0,3$ & 41.667 & 3 & & 31.500 \\
\hline
\end{tabular}




\section{RESULTADOS Y DISCUSIÓN}

Las especies bajo estudio son actualmente "no cultivadas", así como la mayoría de las plantas aromáticas que se venden en las plazas de mercado (Cardona y Barrientos, 2011). Estas son recolectadas del campo en pequeñas cantidades para su venta básicamente como medicina tradicional sin valor agregado en las plazas de mercado como Samper Mendoza en Bogotá. Las aromáticas no cultivadas tienen una tecnología de producción y un mercado poco desarrollados (Barrientos y Cardona, 2010). Las siguientes propuestas se presentan en el marco de este actual escenario.

\section{Costos e ingresos del cultivo}

Los costos de producción del sistema bajo invernadero son 3,3-3,7 veces mayores que el sistema a cielo abierto al inicio. Esto se debe principalmente al costo de la infraestructura de invernadero y al costo del capital financiero, lo que lo convierte en poco atractivo para los pequeños productores. Por otro lado, una mayor producción eleva los costos en mano de obra y transporte. Los costos de mano de obra y tierra son 20 a 40\% más elevados en Bogotá, que en Fusagasugá. En el sistema bajo cubierta el costo de inversión es de 75\%; mientras que en el sistema a cielo abierto está cerca del $40 \%$ del costo total. Los costos de operación alcanzan el 60\%.

El cultivo de Tagetes tiene costos de operación entre 20 y $25 \%$ mayor que el de Lippia, debido a que el primero tiene una mayor densidad de siembra que el segundo (tabla 1). Esto implica un mayor costo en insumos (plantas), mano de obra (plantación, manejo y cosecha) y transporte.

De acuerdo con los vendedores de aromáticas de la plaza de mercado Samper Mendoza de Bogotá, los precios de hierba fresca de las cuatro especies varían, según la oferta y demanda, entre 2.500 y $4.000 \$ / \mathrm{kg}$, siendo el promedio $3.000 \$ / \mathrm{kg}$. A pesar de haber tomado un mismo precio de venta para las cuatro especies, los ingresos anuales por unidad de área varían por efecto del sistema de producción y de cada especie. Los sistemas bajo invernadero, por su mayor número de cosechas al año, generan 33\% más de ingresos que los sistemas a cielo abierto; mientras que las Tagetes, por su mayor densidad de siembra, generan $75 \%$ más ingresos que las Lippia (tabla 6).

El cultivo de las aromáticas bajo estudio requiere, en general, un presupuesto relativamente ele-

Tabla 2. Costos de inversión y operación del cultivo de Lippia bajo invernadero (Bogotá).

\begin{tabular}{|l|c|c|c|}
\hline \multirow{2}{*}{ Factor de producción } & \multicolumn{2}{|c|}{ Costos (\$/ha año) } \\
\cline { 2 - 4 } 1. Tierra & 1.000 .000 & Costo de operación (año 1) & Costo total (año 1) \\
\hline 2. Mano de obra & & 17.565 .000 & 1.000 .000 \\
\hline 3. Insumos & 2.099 .970 & 3.080 .000 & 17.565 .000 \\
\hline 4. Servicios* & 153.189 .800 & 21.432 .372 & 5.179 .970 \\
\hline $\begin{array}{l}\text { 5. Capital } \\
\text { (bienes duraderos)** }\end{array}$ & & & 153.432 .372 \\
\hline $\begin{array}{l}\text { 6. Administración-Seguro- } \\
\text { Impuestos }\end{array}$ & 156.289 .770 & 664.428 & 6600 \\
\hline Total & & 42.741 .800 & 199.031 .570 \\
\hline
\end{tabular}

* Principalmente el servicio de crédito.

** Principalmente el costo del invernadero.

Fuente: elaboración basada en Bandoni (2002); Martínez et al. (2000); 0.J. Rodríguez (comunicación personal, 2011); J. Moreno (comunicación personal, Fresh Andina, 2011). 


\section{Tabla 3. Costos de inversión y operación del cultivo de Lippia a cielo abierto (Fusagasugá).}

\begin{tabular}{|l|c|c|c|}
\hline \multirow{2}{*}{ Factor de producción } & \multicolumn{3}{|c|}{ Costos (\$/ha año) } \\
\cline { 2 - 4 } 1. Tierra & 700.000 & Costo de operación (año 1) & Costo total (año 1) \\
\hline 2. Mano de obra & & 13.925 .000 & 700.000 \\
\hline 3. Insumos & 1.396 .800 & 1.880 .000 & 13.925 .000 \\
\hline 4. Materiales* & & 6.066 .000 & 3.276 .800 \\
\hline 5. Servicios & & 6.256 .988 & 6.066 .000 \\
\hline 6. Capital (bienes duraderos) & 19.360 .000 & 3.300 .000 & 6.256 .988 \\
\hline 7. Administración-Seguro- & & 664.428 & 22.660 .000 \\
\hline Impuestos & & 32.092 .416 & 664.428 \\
\hline Total & 21.456 .800 & & 53.549 .216 \\
\hline
\end{tabular}

* Principalmente Agromulch.

Fuente: elaboración basada en Bandoni (2002); Gitman y Joehnk (2005); Martínez et al.(2000); 0.J. Rodríguez (comunicación personal, 2011); J. Moreno (comunicación personal, Fresh Andina, 2011).

Tabla 4. Costos de inversión y operación del cultivo de Tagetes bajo invernadero (Bogotá).

\begin{tabular}{|l|c|c|c|}
\hline \multicolumn{1}{|c|}{ Factor de producción } & \multicolumn{2}{|c|}{ Costos (\$/ha año) } \\
\hline 1. Tierra & Costo de inversión & Costo de operación (año 1) & Costo total (año 1) \\
\hline 2. Mano de obra & 1.000 .000 & & 1.000 .000 \\
\hline 3. Insumos & & 27.300 .000 & 27.300 .000 \\
\hline 4. Servicios & 3.291 .670 & 3.080 .000 & 6.371 .670 \\
\hline 5. Capital (bienes duraderos) & 153.986 .390 & 22.739 .664 & 22.739 .664 \\
\hline $\begin{array}{l}\text { 6. Administración-Seguro- } \\
\text { Impuestos }\end{array}$ & & & 153.986 .390 \\
\hline Total & 158.278 .060 & 664.428 & 664.428 \\
\hline
\end{tabular}

Fuente: elaboración basada en Serrato (2003); Acosta de la Luz et al. (2001); J. Moreno (comunicación personal, Fresh Andina, 2011$)$; C. Mendoza (comunicación personal, Universidad de Cundinamarca-UDEC, 2011).

Tabla 5. Costos de inversión y operación del cultivo de Tagetes a cielo abierto (Fusagasugá).

\begin{tabular}{|l|c|c|c|}
\hline \multicolumn{1}{|c|}{ Factor de producción } & Costo de inversión & Costo de operación (año 1) & Costo total (año 1) \\
\hline 1. Tierra & 700.000 & & 700.000 \\
\hline 2. Mano de obra & & 20.775 .000 & 20.775 .000 \\
\hline 3. Insumos & 2.588 .500 & 1.880 .000 & 4.468 .500 \\
\hline 4. Materiales & & 6.000 .000 & 6.000 .000 \\
\hline 5. Servicios & 20.891 .390 & 7.648 .280 & 7.648 .280 \\
\hline 6. Capital (bienes duraderos) & & 3.300 .000 & 24.191 .390 \\
\hline $\begin{array}{l}\text { 7. Administración-Seguro- } \\
\text { Impuestos }\end{array}$ & & 664.428 & 664.428 \\
\hline Total & 24.179 .890 & 40.267 .708 & 64.447 .598 \\
\hline
\end{tabular}




\section{Tabla 6. Precios de venta e ingresos generados por el cultivo de Lippia y Tagetes bajo invernadero y a cielo abierto.}

\begin{tabular}{|l|c|c|c|}
\hline Especie, sistema de cultivo y lugar & $\begin{array}{c}\text { Precio unitario } \\
(\$ / \mathrm{kg})\end{array}$ & $\begin{array}{c}\text { Producción promedio anual } \\
\left(\mathrm{kg} \mathrm{ha}^{-1} \mathrm{año}^{-1}\right)\end{array}$ & $\begin{array}{c}\text { Ingresos anuales promedio } \\
(\$ / \text { ha año) }\end{array}$ \\
\hline Lippia bajo invernadero (Bogotá) & 3.000 & 24.000 & 72.000 .000 \\
\hline Lippia a cielo abierto (Fusagasugá) & 3.000 & 18.000 & 54.000 .000 \\
\hline Tagetes bajo invernadero (Bogotá) & 3.000 & 42.000 & 126.000 .000 \\
\hline Tagetes a cielo abierto (Fusagasugá) & 3.000 & 31.500 & 94.500 .000 \\
\hline
\end{tabular}

Fuente: elaboración basada en Díaz (2003) y encuesta vendedores de la plaza de mercado Samper Mendoza, Bogotá (2011).

vado, inclusive a cielo abierto. Esto las convierte en poco competitivas económicamente frente a otras especies de aromáticas; sin embargo, tienen algo positivo, generan elevada demanda de mano de obra. Por su estado actual mayoritariamente silvestre no requieren de un manejo fitosanitario exigente. Tienen de tres a cuatro cosechas por año, pero la producción podría incrementarse si se aumenta la densidad. Sin embargo, hay que mencionar que una mayor oferta también disminuiría los precios de venta afectando así los ingresos.

\section{Rentabilidad del cultivo}

Los VAN (valor actual neto) son positivos, las $\mathrm{R}$ B/C (relación beneficio/costo) están por encima de 1 y las TIR (tasa interna de retorno) por encima de la tasa de descuento aplicada (14\%), excepto en el caso de las Lippia bajo invernadero, cuyo valor de TIR está por debajo de lo mínimo (tabla 7). Los valores varían notablemente por efecto del sistema de producción y de la especie. Los sistemas a cielo abierto, tanto en Lippia como en Tagetes, son 5 a 10 veces más rentables (TIR) que los sistemas bajo invernadero. Y los Tagetes son aproximadamente de 2 a 3 veces más rentables que las Lippia. La baja rentabilidad de las Lippia bajo invernadero puede pasar a ser aceptable si, por ejemplo, se incrementa el rendimiento del cultivo. Esto puede lograrse incrementando la densidad de la plantación (ahora $1 \times 0,5 \mathrm{~m}$ ).

\section{Costos e ingresos de la producción de aceites esenciales}

El sistema de extracción de aceites esenciales utilizado en la investigación, y el más común en la industria, es el de arrastre con vapor. Se utiliza un equipo de tamaño mediano que procesa 600 $\mathrm{kg}$ de materia prima (material fresco) en aproximadamente 7 horas (tabla 8).

En los costos de extracción de aceites esenciales, la materia prima (insumos) participa con más del $90 \%$ de los costos totales, el capital con 3\%,

\section{Tabla 7. Rentabilidad del cultivo de Lippia y Tagetes bajo invernadero y a cielo abierto.}

\begin{tabular}{|l|c|c|c|}
\hline Especie, sistema de cultivo y lugar & R B/C & VAN & TIR (\%) \\
\hline Lippia bajo invernadero (Bogotá) & 1,13 & 21.014 .172 & 8,44 \\
\hline Lippia a cielo abierto (Fusagasugá) & 5,58 & 94.979 .106 & 31,70 \\
\hline Tagetes bajo invernadero (Bogotá) & 2,19 & 187.937 .220 & 159,60 \\
\hline${ }^{1}$ Tagetes a cielo abierto (Fusagasugá) & 9,34 & 195.816 .488 & \\
\hline
\end{tabular}

${ }^{1}$ La especie Tagetes zypaquirensis, dadas sus características, no se cultiva en clima cálido, por lo cual no se evidencian sus rendimientos para el escenario de invernadero en Fusagasugá.

Fuente: elaboración basada en Bandoni (2002); MADR (2009); Serrato (2003); Acosta de la Luz et al. (2011); 0.J. Rodríguez (comunicación personal, 2011); J. Moreno (comunicación personal, Fresh Andina, 2011); C. Mendoza (comunicación personal, Universidad de Cundinamarca-UDEC, 2012) y encuesta vendedores de la plaza de mercado Samper Mendoza, Bogotá (2011). 
Tabla 8. Costo del proceso de extracción de aceites esenciales ${ }^{1}$ (año 1).

\begin{tabular}{|l|c|c|c|}
\hline Factores de producción & Costo de inversión (\$) & $\begin{array}{c}\text { Costo de operación } \\
(\$ \text { año 1) }\end{array}$ & Costo total (\$año 1) \\
\hline Mano de obra & & 25.200 .000 & 25.200 .000 \\
\hline Insumos & & 879.840 .000 & 879.840 .000 \\
\hline Equipos e infraestructura ${ }^{2}$ & 30.000 .000 & & 30.000 .000 \\
\hline Herramientas & 115.000 & 65.258 & 115.000 \\
\hline Servicios & & & 65.258 \\
\hline TOTAL & 30.115 .000 & 905.105 .258 & 935.220 .258 \\
\hline
\end{tabular}

${ }^{1}$ Por día se realizan dos ciclos de producción (600 kg/ciclo), 14 horas y se procesan $1.200 \mathrm{~kg}$ de materia prima. Se trabajan $5 \mathrm{~d}$ a la semana, 4 semanas al mes y 12 meses al año; de lo cual resulta: $240 \mathrm{~d}$ de trabajo por $1.200 \mathrm{~kg}$ de material fresco procesado por día $=288.000 \mathrm{~kg}$ de materia prima procesada al año (Personero Green Andina, comunicación personal, 2011).

${ }^{2}$ El extractor cuesta $\$ 26$ millones y la infraestructura \$4 millones. Su vida útil es de 10 años.

Fuente: elaboración basada en Sánchez (2006); D. Barrera (comunicación personal, 2011); I. Rivero (comunicación personal, Green Andina, 2011); F.

Sánchez (comunicación personal, Green Andina, 2011).

\section{Tabla 9. Superficie de cultivo mínima requerida para satisfacer la capacidad de procesamiento del extractor} (288 t año $\left.{ }^{-1}\right)$.

\begin{tabular}{|l|c|c|}
\hline \multicolumn{1}{|c|}{$\begin{array}{c}\text { Especie, sistema de cultivo y } \\
\text { lugar }\end{array}$} & $\begin{array}{c}\text { Producción promedio anual de materia } \\
\text { prima }\left(\mathrm{kg} \mathrm{ha}^{-1} \mathrm{añ}^{-1}\right)\end{array}$ & $\begin{array}{c}\text { Superficie de cultivo mínima requerida } \\
\text { (ha) }\end{array}$ \\
\hline Lippia bajo invernadero (Bogotá) & 24.000 & 12,0 \\
\hline Lippia a cielo abierto (Fusagasugá) & 18.000 & 16,0 \\
\hline Tagetes bajo invernadero (Bogotá) & 42.000 & 6,9 \\
\hline $\begin{array}{l}\text { Tagetes a cielo abierto } \\
\text { (Fusagasugá) }\end{array}$ & 31.500 & 9,1 \\
\hline
\end{tabular}

y la mano de obra solo con aproximadamente el $2 \%$. El costo de inversión en este negocio es relativamente bajo (3\%), mínimo frente al costo de operación.

Para que un equipo mediano de extracción de aceites pueda funcionar a su entera capacidad se requieren superficies de cultivo entre 7 y 16 ha. Dado que un pequeño productor no podría financiar un cultivo de ese tamaño, puede ser una oportunidad para organizaciones de pequeños productores o empresarios medianos del agronegocio de aromáticas.

El rendimiento de aceites esenciales es en su mayoría satisfactorio, siendo mayor en las Lippia que en las Tagetes. El contenido de aceites esenciales es mayor en cultivos de lugares cálidos como Palmira, que de lugares templados o fríos como Fusagasugá o Bogotá (tabla 10). El precio de los aceites depende del quimiotipo que contiene. El quimiotipo Timol (tabla 11) parece ser el más apetecido por el mercado, pues recibe los mejores precios. El precio de los aceites de las Tagetes es evidentemente más bajo que el de las Lippia debido a que sus quimiotipos y ellas mismas, como especie, son poco conocidas.

\section{Rentabilidad de la producción de aceites esen- ciales}

La extracción de aceites esenciales de Lippia origanoides muestra una rentabilidad bastante atractiva (tabla 12) al igual que Lippia alba en el escenario de libre exposición; mientras que las de Tagetes presenta pérdidas (VAN negativa y $\mathrm{R}$ 
B/C menor a 1). El hecho de que las Lippia tengan una TIR bastante elevada se debe a que los costos de inversión frente a los costos de operación anual son mucho menores. Esta situación se supera con la TIR modificada (tabla 12).

\section{CONCLUSIONES}

El cultivo de las especies bajo estudio, excepto de Lippia bajo invernadero, tienen ren- tabilidades altas. Las Tagetes, por su mayor densidad de siembra, son más rentables que las Lippia.

Los costos de producción de estos cultivos son relativamente elevados frente a otras especies de hortalizas, especialmente cuando están bajo invernadero. Esto puede ser una limitante para los pequeños productores, y más aún si a consecuencia de un incremento de la oferta, los precios de venta bajan.

Tabla 10. Quimiotipos y rendimiento de producción de aceite (\%).

\begin{tabular}{|c|c|c|c|c|c|c|c|c|c|c|}
\hline \multirow[t]{2}{*}{ Especies } & \multirow[t]{2}{*}{ Quimiotipos } & \multicolumn{3}{|c|}{ Rendimiento (\%) } & \multicolumn{3}{|c|}{$\begin{array}{l}\text { Cantidad de aceite crudo } \\
\text { (L) por } 1.000 \mathrm{~kg} \text { de } \\
\text { materia prima }\end{array}$} & \multicolumn{3}{|c|}{$\begin{array}{l}\text { Cantidad de aceite crudo } \\
\qquad\left(\mathrm{L} \mathrm{ha}^{-1} \mathrm{año}^{-1}\right)^{2}\end{array}$} \\
\hline & & BTA & FUSA & PAL & BTA & FUSA & PAL & BTA & FUSA & PAL \\
\hline Lippia alba & $\begin{array}{l}\text { Limoneno (49- } \\
55 \%) \text { Carvona } \\
(20-25 \%)\end{array}$ & 0,15 & 0,60 & 0,77 & 1,50 & 6,00 & 7,70 & 36,00 & 108,00 & 270,00 \\
\hline $\begin{array}{l}\text { Lippia } \\
\text { origanoides }\end{array}$ & Timol (60\%) & 0,81 & 1,16 & 3,55 & 8,10 & 11,60 & 35,50 & 194,40 & 208,80 & 639,00 \\
\hline $\begin{array}{l}\text { Tagetes } \\
\text { caracasana }\end{array}$ & $\begin{array}{l}\text { Trans-ocimenona } \\
(49,3-53,2 \%)\end{array}$ & $0,14^{1}$ & NA & NA & 1,40 & NA & NA & 58,80 & NA & NA \\
\hline $\begin{array}{l}\text { Tagetes } \\
\text { zypaquirensis }\end{array}$ & $\begin{array}{l}\text { Monoterpenoides } \\
(41 \%) \\
\text { Dihidrotagenona } \\
(30 \%)\end{array}$ & 0,20 & NA & 0,12 & 2,00 & NA & 1,20 & 84,00 & NA & $50,40^{3}$ \\
\hline
\end{tabular}

${ }^{1}$ El rendimiento oscila entre $0,12 \%$ y $0,16 \%$, de cuyo rango se toma la moda del rendimiento. El rendimiento se mide en litros de aceite por cada $100 \mathrm{~kg}$ de materia prima (M.I. Chacón, comunicación personal, 2012).

${ }^{2}$ Se toman los rendimientos anuales de producto fresco por hectárea (tabla 1) de los dos sistemas: a cielo abierto (menor) y bajo invernadero (mayor).

${ }^{3}$ Se toma el dato de rendimiento anual de producto en fresco por hectárea correspondiente al sistema bajo invernadero.

BTA: Bogotá, FUSA: Fusagasugá, PAL: Palmira.

Fuente: elaboración basada en Blanco y Agudelo (2007); M.I. Chacón (comunicación personal, 2011).

\section{Tabla 11. Precios de los aceites esenciales comerciales.}

\begin{tabular}{|c|c|}
\hline Especies & Precio de $1 \mathrm{~L}$ de aceite crudo a nivel productor (\$) \\
\hline Lippia alba & 1.225 .000 \\
\hline Lippia origanoides $^{1}$ & 2.925 .000 \\
\hline Tagetes caracasana $^{2}$ & 784.448 \\
\hline Tagetes zypaquirensis $^{2}$ & 784.448 \\
\hline
\end{tabular}

1 Este aceite no se comercializa en la actualidad, por esta razón se toma como referencia el aceite esencial de tomillo ya que entre sus componentes principales se encuentra el quimiotipo Timol.

${ }^{2}$ Este aceite no se comercializa en la actualidad, por lo cual se toma como referencia el aceite esencial de ylang-ylang por su similitud de compuestos. Fuente: elaboración basada en I. Rivero (comunicación personal, Green Andina, 2011). 
Tabla 12. Rentabilidad anual de la producción de aceite esencial de Lippia y Tagetes.

\begin{tabular}{|c|c|c|c|c|c|c|c|c|c|c|c|c|}
\hline \multirow[t]{2}{*}{ ESPECIES } & \multicolumn{3}{|c|}{$\mathrm{RB} / \mathrm{C}$} & \multicolumn{3}{|c|}{ VAN } & \multicolumn{3}{|c|}{ TIR (\%) } & \multicolumn{3}{|c|}{ TIR MODIFICADA $(\%)^{1}$} \\
\hline & BTA & FUSA & PAL & BTA & FUSA & PAL & BTA & FUSA & PAL & BTA & FUSA & PAL \\
\hline Lippia alba & $-49,62$ & 165,80 & 247,00 & -1.525 .759 .819 & 4.969 .390 .350 & 7.423.113.747 & $\mathrm{NA}$ & 2.621 & 3.915 & $\mathrm{NA}$ & 90,02 & 97,77 \\
\hline $\begin{array}{l}\text { Lippia } \\
\text { origanoides }\end{array}$ & 804,70 & $1.204,96$ & $3.937,84$ & 24.225.080.440 & 36.287 .502 .181 & 118.656 .610 .640 & 12.771 & 19.130 & 62.549 & 122,56 & 131,73 & 160,87 \\
\hline $\begin{array}{l}\text { Tagetes } \\
\text { caracasana }\end{array}$ & $-78,52$ & NA & NA & -2.396 .814 .304 & NA & $N A$ & NA & NA & NA & NA & NA & NA \\
\hline $\begin{array}{l}\text { Tagetes } \\
\text { zypaquirensis }\end{array}$ & $-60,12$ & $N A$ & $-84,65$ & -1.842 .244 .774 & NA & -2.581 .670 .814 & $\mathrm{NA}$ & $N A$ & NA & $N A$ & NA & $\mathrm{NA}$ \\
\hline
\end{tabular}

${ }^{1}$ Se utiliza la TIR modificada para solucionar problemas de TIR múltiples y para eliminar el supuesto de la TIR de reinvertir todos los flujos del proyecto a esa tasa, por lo cual se tiene un flujo de fondos que es inversión en el período inicial (año 0), luego 0 en todos los otros periodos de tiempo y finalmente un valor acumulado en el último periodo. Al calcular la TIR de ese flujo de fondos se obtiene un parámetro de rentabilidad más real (Besley and Brigham, 2008)

BTA: Bogotá, FUSA: Fusagasuga, PAL: Palmira.

Fuente: elaboración basada en Serrato-Cruz et al. (2008); Sánchez (2006); I. Rivero (comunicación personal, Green Andina, 2011). 
Estos cultivos son viables económicamente solo si tienen un mercado diferente al actual, que es un mercado tradicional, donde la demanda es muy baja. Su viabilidad implicaría hacer un trabajo extra de cooperación vertical con la industria de aceites.

En Colombia ya está establecida una industria de extracción de aceites esenciales, pero para poder trabajar con Lippia y Tagetes se requiere de materia prima en cantidades que actualmente no se ven en el mercado local. Actualmente las Lippia y Tagetes que llegan al mercado en su mayoría se recolectan del campo, no se cultivan.

Los costos de inversión para una extractora mediana no son elevados, lo más costoso (>90\%) es la materia prima. Para que una extractora de 600 $\mathrm{kg}$ de capacidad funcione a su capacidad "normal", se necesitan entre 7 y 16 ha de cultivo, lo que podría ser una oportunidad para empresarios medianos o para asociaciones de pequeños productores.

El rendimiento de aceites en Lippia es apreciable (alrededor de 1\%). Las zonas cálidas tienen los mejores rendimientos. Por el contrario, el rendimiento de aceite de Tagetes es relativamente bajo $(<0,5 \%)$. Esto sumado a su menor precio de venta hace que la extracción de aceites de $T$. caracasana y $T$. zypaquirensis, por el momento, no sean rentables.
Los aceites de las especies bajo estudio son poco conocidos en el mercado. Esto hace presumir que al principio su precio sea menor al presentado en este trabajo. Por ser productos nuevos, la venta de estos aceites requeriría de un trabajo adicional de mercadeo, que puede comenzar en los nichos de los quimiotipos conocidos, como timol y limoneno.

El manejo del cultivo de estas especies no es suficientemente conocido, los datos son aún referenciales, por lo que se recomienda realizar ensayos durante todo el año para recoger información agronómica, especialmente relacionada con las densidades, la cosecha, rendimiento y renovación de plantación.

Si se pone en funcionamiento un extractor mediano de aceite para Tagetes o Lippia, se recomienda tener suficiente materia prima, aproximadamente $300 \mathrm{t}$ anuales (7 a 16 ha de cultivo), para poder trabajar continuamente.

La iniciativa de industrializar estas nuevas especies para obtención de aceites esenciales debe ir acompañada de un proyecto integral que contemple producción empresarial o cooperativa, y encadenamiento con la industria para la transformación y comercialización.

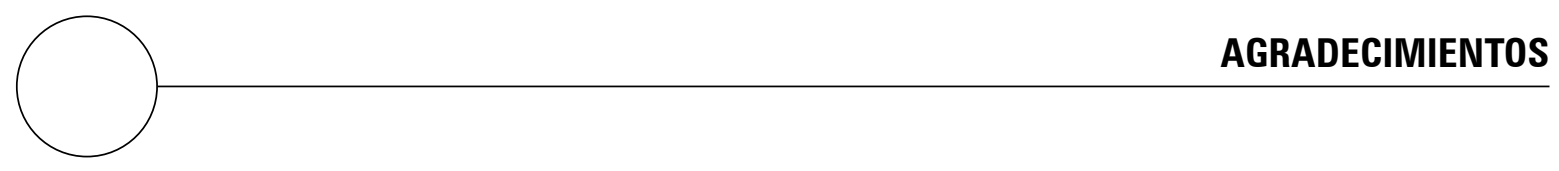

La presente investigación fue financiada por el Ministerio de Agricultura y Desarrollo Rural (MADR) de Colombia, contrato 064-2007V7163-50-07 y la Dirección de Investigaciones de la Universidad Nacional de Colombia, sede Bogotá. Los investigadores agradecen la colaboración de las empresas Green Andina Ltda. y Fresh Andina por la valiosa información aportada a la investigación, así como al personal técnico y científico del proyecto. 


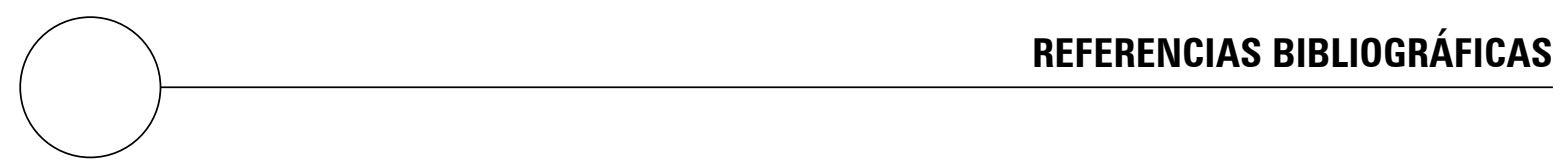

Acosta de la Luz, L., I. Echevarría, C. Rodriguez y M. Milanés. 2011. Momento óptimo de plantación y de cosecha de Tagetes lucida Cav. Rev Cubana Plant. Med. 16(2), 201-208.

Bandoni, A.L. (ed.). 2002. Los recursos vegetales aromáticos en Latinoamérica. Editorial de la Universidad Nacional de la Plata, Buenos Aires.

Bareño, P. y J. Clavijo. 2006. Hierbas aromáticas culinarias para exportación en fresco. pp. 7-9. En: Clavijo, J., P. Bareño, L. Chaparro y C. Guío (eds.). Últimas tendencias en hierbas aromáticas culinarias para exportación en fresco. Produmedios, Bogotá.

Barrientos F., J.C. y J.O. Cardona. 2010. Los pequeños productores están limitados para adoptar nuevos cultivos. El caso de las hierbas aromáticas en la región de Sumapaz, Cundinamarca. Agron. Colomb. 28(1), 99-106.

Barrientos, J.C. y M.L. Reina. 2012. Evaluación del potencial económico y de mercado de 4 especies aromáticas promisorias para producir aceites esenciales de uso agrícola en Colombia (Lippia alba, Lippia origanoides, Tagetes caracasana y Tagetes zypaquirensis). Informe del Proyecto Evaluación fenotípica, química y agronómica de especies nativas y foráneas promisorias por calidad de aceites esenciales $y$ con uso potencial en el sector agrícola. Facultad de Agronomía, Universidad Nacional de Colombia, Bogotá.

Biocomercio Sostenible. 2003. Estudio del mercado colombiano de aceites esenciales. Instituto de Investigación de Recursos Biológicos Alexander von Humboldt, Bogotá.

Blanco, K. y A. Agudelo. 2007. Estudio comparativo de los aceites esenciales de Lippia alba Mill N.E. Brown ex Britton \& Wills cultivada con tres tipos de compost.Trabajo de grado. Universidad Industrial de Santander, Bucaramanga, Colombia.

Besley and Brigham. 2008. Principles of finance. $4^{\text {th }}$ ed. South-Western College Pub.; Mason, $\mathrm{OH}$.

Cardona, J.O. y J.C. Barrientos F. 2011. Producción, uso y comercialización de especies aromáticas en la región Sumapaz, Cundinamarca. Rev. Colomb. Cienc. Hortíc. 5(1), 114-129.

Colombian National Press. 2006. Crece la demanda por productos con sello natural, a nivel mundial. Por- tafolio Comercio Exterior de Colombian National Press, Bogotá. 10 de noviembre de 2006.

Díaz, J.A. 2003. Informe técnico. Caracterización del mercado colombiano de plantas medicinales y aromáticas. Instituto Alexander von Humboldt y Ministerio de Ambiente, Vivienda y Desarrollo Territorial, Bogotá.

Díaz, J.A. 2006. Estrategia para tres sectores de biocomercio con estudios de mercado específicos. Volumen VII. Corporación Andina de Fomento e Instituto Alexander von Humboldt, Bogotá.

Fundacofan. 2004. Estudio internacional de mercado de plantas medicinales, aromáticas, condimentarias y sus subproductos. Fundación Colombiana para la Farmacia Natural, Cali.

Gitman, L. y M. Joehnk. 2005. Fundamentos de inversión. Editorial Pearson Education S.A., Madrid.

MADR. 2009. Agenda prospectiva de investigación y desarrollo tecnológico para la cadena productiva de plantas aromáticas, medicinales, condimentarías y afines en ingredientes naturales para la industria cosmética en Colombia. Ministerio de Agricultura y Desarrollo Rural, Bogotá.

Martínez, J.V., H.Y. Bernal y A. Cáceres (eds.). 2000. Fundamentos de agrotecnología de cultivo de plantas medicinales Iberoaméricanas. Convenio Andrés Bello (CAB) y Programa Iberoamericano de Ciencia y Tecnología para el Desarrollo (CYTED), Bogotá.

Ministerio de Agricultura y Desarrollo Rural, Instituto Alexander von Humboldt Colombia y Cámara de Comercio de Bogotá. 2008. Definición de la agenda prospectiva de investigación para la cadena productiva de plantas aromáticas, medicinales y condimentarias y afines con énfasis en ingredientes naturales para la industria cosmética en Colombia. Informe final: Análisis de desempeño, implementación de las herramientas del sistema de inteligencia tecnológica, análisis prospectivo. Bogotá.

Pacheco, A. y J. Pohlan. 2006. Efectos de plantas aromáticas sobre la estimulación de crecimiento y rendimiento en cultivos tropicales. pp. 16-20. En: Clavijo, J., P. Barreño, L. Chaparro y C. Guío (eds.). Últimas tendencias en hierbas aromáticas culinarias para exportación en fresco. Produmedios, Bogotá. 
Repetto, H. 2005. La cadena productiva de las plantas aromáticas, medicinales y condimentarías (PAM) en Cundinamarca. Asociación Colombiana para el Avance de la Ciencia (ACAC), Bogotá.

Sánchez, F. 2006. Extracción de aceites esenciales. Experiencia colombiana. pp.1-8. En: Memorias II Segundo Congreso Internacional de Plantas Medicinales y Aromáticas. Universidad Nacional de Colombia, Palmira, Colombia.
Serrato, M. 2003. Aspectos del cultivo de dos especies de tagetes productoras de aceites esenciales. Naturaleza y Desarrollo 1(1), 15-22.

Serrato-Cruz, M.A., F. Díaz-Cedillo y J.S. BarajasPérez. 2008. Composición en el aceite esencial en germoplasma de Tagetes filifolia Lag. de la región centro-sur de México. Agrociencia 42, 277-285. 